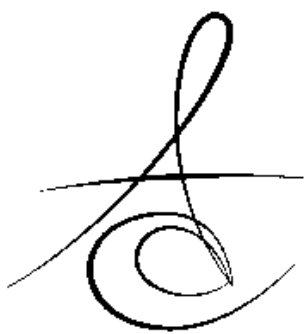

\title{
TAM-ARK İMPLANT-DESTEKLİ MONOLİTİK ZİRKONYA SABİT DENTAL PROTEZLER: LITERATÜR DERLEMESİ
}

\section{COMPLETE-ARCH IMPLANT-SUPPORTED MONOLITHİC ZIRCONIA FIXED DENTAL PROSTHESES: A SYSTEMATIC REVIEW}

\author{
Doç. Dr. Rukiye DURKAN* \\ Dr. Öğr. Üyesi Gonca DESTE* \\ Doç. Dr. Perihan OYAR ${ }^{* *}$
}

Makale Kodu/Article code: 2987

Makale Gönderilme tarihi: 09.08.2016

Kabul Tarihi; 30.09.2016

\section{öz}

Tam dişsizlik vakalarında implant destekli monolitik zirkonya sabit dental protezlerin yapımları giderek artmaktadır. Hekim-hasta bakış açısıyla ve teknik bakımlardan yüksek kalitede restorasyonlar elde edildiği gözlenmektedir. Son teknoloji ürünü bilgisayar destekli tasarım ve bilgisayar destekli üretim (BDT/BDÜ) (computer aided design/ computer assisted manufacture-CAD/CAM) ile elde edilen implant üstü monolitik zirkonya restorasyonlarda estetik ve yüksek dayanıklılık özellikleri birlikte sunulmaktadır.

Bu derlemede, öncelikle tam-ark implant-destekli sabit protez tipleri ile ilgili genel bir değerlendirme yapıldı. Takiben komple-ark implant-destekli monolitik sabit dental protezlerin (complete-arch implant-supported fixed dental prostheses) uygulamalarına yönelik olan çalışmaların ışığı altında, avantaj ve dezavantajları değerlendirildi. Sonuç olarak, tam ark implant destekli monolitik zirkonya sabit protezlerle ilgili olarak yapılan klinik çalışmaların kısa dönem (5-10 yıl arası) başarı oranının çok yüksek olduğu ancak uzun dönem (10 yıldan fazla) sonuçlarının yeterli olmadığı, klinik performanslarının henüz tam olarak netlik kazanmadığı, bu yönde daha fazla çalışma yapılması gerekliliği tespit edildi.

Anahtar Kelimeler: Monolitik zirkonya seramik sistemleri, tam ark implant destekli sabit protezler, zirkonya

\section{ABSTRACT}

Fixed dental prostheses are produced from gradational in edentulous patient cases, it is observed that implant supported monolithic zirconia fixed dental prostheses constructions are ever increased. High quality restorations are achieved in the aspect of clinicianpatient and technical maintenance. Especially, high durability and aesthetic properties are presented together in monolithic implant restorations produced with the latest technology computer aided design/ computer assisted manufactured (CAD/CAM).

In this review, primarily an assessment made about complete arch implant supported fixed prostheses types. Then, the advantage and disadvantages were summarized in the lights of the studies of completearch implant-supported fixed dental prostheses. Consequently, it was determined that success rate were very high in short term clinical studies about complete-arch implant-supported fixed dental prostheses (5-10 years). However, long term (more than 10 years) study results were limited, to bring a conclusion and clinical performances are not yet fully clear. Thus, it is necessary to carry out further studies and wait the long term prognosis of this type of restorations in long term studies.

Keywords: Monolithic zirconia ceramic systems, full arch implant supported fixed prostheses, zirconia

\footnotetext{
${ }^{*}$ Afyon Kocatepe Üniveritesi Diş Hekimliği Fakültesi, Protetik Diş Tedavisi AD, Afyon.

**Hacettepe Üniversitesi Sağlık Hizmetleri Meslek Yüksekokulu, Diş Protez Teknolojileri Programı, Ankara
} 


\section{GİRIŞ}

Diş hekimliğinde, tam dişsiz hastaların protetik tedavilerinde osteointegre implantların kullanılması kabul edilen bir tedavi türüdür. ${ }^{1}$ Tedavi planlaması ile ilgili en önemli konular vertikal ve horizantal doku kaybının sınırları, implant pozisyonları ve protez tipi olarak sayılabilir. ${ }^{1}$ Fonksiyon, fonasyon, estetik ve gingival mimariyi oluşturmak için metal-seramik, metal-akrilik, metal-kompozit, zirkonya-seramik ve monolitik zirkonya sistemler kullanılmaktadır. ${ }^{2-5}$ Alt çene ve/veya üst çene tam dişsiz vakaların rehabilitasyonlarında, tam ark implant-destekli monolitik zirkonya sabit dental protezlerin (monolithic zirconia fixed dental prosthesis) yapımı en yeni teknoloji ve yeni jenerasyon materyallerin kullanımı ile mümkün olmaktadır., ${ }^{6,7}$ Ayrıca tam ark implant-destekli restorasyonlarda vaka raporlarında ve 5-10 yıllık klinik takip çalışmalarında yüksek başarı oranları belirtilmektedir.,3-10 Tam dişsizlik vakalarında estetik, hijyen ve dayanıklılık bakımından implant destekli sabit protezler önerilmektedir. Bu protezlerde, mandibulada 10 yıllık sağ kalım oranı \%97 ve maksillada 5 yıllık sağ kalım oranı \%95 iken her iki çene için başarı oranı \%90 olarak verilmektedir. ${ }^{11-13}$

1980'li yılların sonlarında tüm dünyada bilgisayar destekli tasarım ve bilgisayar destekli üretim (BDT/BDÜ) sistemlerinin yaygınlaşmasına paralel olarak tam seramik sistemlerinin kullanımı artmıştır. ${ }^{14}$ Monolitik seramik sistemleri yüksek dayanıklılık, yüksek translusent özelliği ve ideal estetik sağlaması ile günümüzde yaygın hale gelmiştir. ${ }^{15} \mathrm{Bu}$ derlemenin amacı, tam dişsizlik olan hasta grubunda, tam ark implant uygulamaların takiben implant üstü sabit protezlerde kullanılan üst yapı restorasyonları ile bunların avantaj ve dezavantajlarını incelemektir. Bunun yanında, tam ark implant üstü monolitik zirkonya sabit protezlerin hasta-hekim ve teknik uygulama yönünden avantaj ve dezavantajların incelemek ve gelinen son noktada kullanım önerilerinde bulunmaktır.

Tam-ark implant-destekli sabit dental protezler

\section{Metal-destekli seramik sabit protezler}

Metal destekli seramik restorasyonlar protetik diş hekimliğinde uzun yıllar altın standart olarak kabul edilmiştir ve implant üstü sabit protez materyalleri olarak kullanılmıştır. ${ }^{16,17} \mathrm{Bu}$ tür restorasyonların avantajları: estetik olması, dayanıklıııları nedeni ile ön ve arka grup restorasyonlarda birlikte kullanılması, laboratuar işlemlerinin iyi bilinmesi ve kolay olması, maliyetinin düşük olması kolay temizlenebilmesi, sökülmesi ve simantasyonun kolay olması, pek çok klinik ve in vitro çalışmada $10-11$ yıllık \%95 başarı oranının belirtilmesidir. ${ }^{18-21}$ Dezavantajları ise, metal bir alt yapı ve üzerine uygulanan seramik arasında bağlantı problemlerinin olması, seramik üst yapıda ciping olması, metal altyapı nedeni ile özellikle servikal bölgelerde dişeti bölgesinde gri metal yansıması ile estetik kayıplar, nikel içerikli metal kullanımlarında \%10-15 hasta grubunda metal alerjisine bağlı ağız ve dişeti reaksiyonları, kıymetli metal altyapı kullanımlarında maliyetin yükselmesi, laboratuvar işlemlerinin yeni sistemlere göre daha uzun sürmesi, daha fazla zaman olması ve yeni sistemlere göre estetik ve dayanıklılık özelliklerinin daha düşük olması şeklinde sıralanabilir. ${ }^{8,22-26}$ Özellikle son yıllarda hastaların daha estetik ve daha dayanıklı restorasyonlar talep etmeleri ve teknolojik gelişmelerin artması ile birlikte bu avantajlara sahip üstün özellikte sistemler üretilmektedir. ${ }^{27}$

\section{Metal-destekli hibrit sabit protezler}

$15 \mathrm{~mm}$ ve daha fazla kron yüksekliği mesafesi olan durumlarda sabit protezlere alternatif olarak sabit hibrit protezler yapılabilir. Metal altyapı üzerinde akrilik, porselen ve kompozit rezin dişlerin, kaide olarak akrilik rezinin kullanımı ile uygulanır. Hem alt hem üst çene tam dişsizlik vakalarında 6-8 implant üzerine vida tutuculu hibrit protezler yapılmaktadır. ${ }^{28,29}$ Bu yöntemin avantajları; hastaya komple sabit protez sunması, daha az implant sayısı ve ekonomik olması, özellikle maksiller sinüs ogmentasyonu ve yönlendirilmiş doku rejenerasyonu komplikasyonlarından kaçınılmasıdır. Hibrit protezlerle kısa dönem başarılı sonuçlar bildirilmiştir. ${ }^{30-32}$ Papapyridakos ve ark ${ }^{33}$ implant-destekli tam ark sabit hibrit protezlerin teknik ve biyolojik komplikasyonlarına yönelik yaptıkları derlemede; implant çevresi kemik kaybı; 5 yıl sonunda 2 mm'den fazla ve kayıp hızı \%20.1 iken, 10 yıl sonunda \%40.3 olmaktadır. Vida gevşemesi ve kırılması gibi teknik problemlerin oranı 5 yıl sonunda $\% 10.4$ ve 10 yıl sonunda \%20.8 olmaktadır. ${ }^{33}$ Veneer materyalde kırılma, ciping oranı 5 yıl sonunda \%30.3, 10 yıl sonunda \%66.6 oranı ile yaygın gözlenmektedir. ${ }^{33}$ Kwon ve ark ${ }^{30}$ tarafından yapılan bir derlemede, kısa dönemde 18 adet tam-ark sabit 
implant destekli hibrit protez ve implant destekleri incelenmiştir. 12 makalenin kısa dönem sonuçlarına göre sağ kalım protezler için \%93.3-100 ve implantlar için \%87.89-100 olarak bildirilmiştir. ${ }^{30}$ Uzun dönem çalışmalarda, başarı oranlarının düşük olmasının implant yüzey modifikasyonlarının olmamasından kaynaklanabileceği düşünülmekte ve günümüz implant sistemlerindeki gelişmelere paralel olarak son dönemlerde yapılan ve kısa dönem sonuçları verilen çalışmalarda başarı oranları daha yüksek olarak bildirilmektedir. ${ }^{30}$

Metal destekli-akrilik rezin hibrit köprüler, özellikle kemik rezorbsiyonunun yüksek olduğu üst ve/veya alt çene implant destekli tam ark restorasyonlardır. Çenelerde metal destekli seramik restorasyon yapımını engelleyecek derecede kemik kaybı varsa ve kemik ogmentasyonu uygulanmamışsa, bu tip hibrit protezler yapılmaktadır. Metal altyapı üzerine akrilik rezin dişlerin yerleştirildiği ve servikal bölgede pembe akrilikten dişeti ve kaybedilen mukozayı içeren protezlerdir. ${ }^{4,30,34,35}$ Bu köprülerin avantajları; özellikle kayıp kemik ve mukozanın akrilik rezin materyali ile telafi edilmesiyle estetik olarak hastaların memnun olması, ağız çevresi dudak ve yanak desteğinin sağlanması, implant üzerine vidalı sistem olduğu için hekim tarafindan çıkarılarak implant ve protezin mekanik, biyolojik ve hijyenik kontrollerinin rahatlıkla yapılması, tamir edilmesinin kolay olmasıdır. ${ }^{2}$ Ancak dezavantajlarl; cerrahi tekniklerdeki gelişmeler sayesinde kret ogmentasyonlarının yapılabilmesi ile büyük hacimlere sahip olan hibrit protezlerin tercih edilmemesi, akrilik diş ve dişeti kullanımasına bağı olarak akrilik rezin materyal dezavantajlarına bağı materyalin dayanıklıı̆ının düşük olması, estetik olmaması, renklenme ve zamanla su emilimine bağı deformasyonların olması, akrilik dişlerde kırılmalar, renklenmeler ve aşınmalar estetik olumsuzluklar oluşturmaktadır. ${ }^{35-39}$ On yıllık kullanımda en az 5-6 defa akrilik kısımların yenilenmesi, hacimli protezlerin hastaları rahatsı etmesi, hijyenik olmaması, protezimplant bağlantı vidalarında gevşeme ve kırılmalar, protez metal altyapısında kırımalar olması ve uzun dönem kullanımda gingival, estetik ve fonksiyonel başarısızı|ılardır. ${ }^{37-42}$

Metal destekli-kompozit rezin hibrit köprüler, akrilik rezin köprülere alternatif olarak kullanılmıştır. Yeni nesil kompozit rezinlerin aşınma direnci, renklenme direnci ve estetik özellikleri akrilik rezinlere oranla daha yüksektir. ${ }^{2,20}$ Ancak bu restorasyonlarda da servikal bölgede renklenme estetik bozulmalar ve dayanıklığın metal-seramik restorasyonlardan daha düşük olması dezavantajları bulunmaktadır. Ayrıca in vivo ve iv vitro çalş̧maların yetersizliği gözlenmektedir. ${ }^{2,43}$ Metalik alt yapılar, büyük hacimli ve ağır olmakta, döküm ve politür işlemleri zor olmaktadır. ${ }^{44}$

\section{Zirkonya-destekli seramik sabit} protezler

Son 20 yıldır zirkonya altyapı seramik sistemleri ile bilgisayar destekli tasarım ve bilgisayar destekli üretim (computer-aided design and computer-aided manufacturing (CAD/CAM)) yapılan restorasyonların gelişmesi ve zirkonya kor seramik sistemlerinin bu yöntemler ile üretilmesi ile daha hızlı, daha estetik ve dayanıklı protezler yapılmaktadır. ${ }^{45} \mathrm{Bu}$ protezlerin avantajlarl; metal alt yapının yerine diş renginde olan zirkonya kor yapının gelmesi ile estetik, hijyen ve dişeti sağlığının daha iyi bir duruma getirilmesidir. Bilgisayar destekli olması ile hassas ve teknik olarak uyumlu olması, kısa laboratuar işlemleri ve hastaya teslim aşamalarının kısa olması, psikolojik olarak hastaların metal isteksizliğini elimine etmesi sayılmaktadır. ${ }^{45,46}$ Dezavantajları; 0.5-0.7 mm kalınlı̆ında hazırlanan zirkonya alt yapılarda kırılmalar olması, üst yapının farklı seramikten yapılması ile gelişen bağlantı sorunları, zirkonya kor-seramik üst yapı termal genleşme katsayı uyumsuzlukları, seramik üst yapıda kırımalar, ciping ve ayrımalar olmasıdır. Kısa dönem klinik verilerin olumlu olduğu belirtilmektedir. ${ }^{47-50}$ Mandibular tam ark zirkonya destekli 9 sabit protezde 8 yillık takip sonunda hiçbir protezde gövde kırılması oluşmadığı ancak \%40 veneer seramik kırımaları olduğu buna rağmen hastaların memnun olduğu protezler olarak rapor edilmişlerdir. ${ }^{3}$ Maksiller/mandibuler tam ark zirkonya destekli 16 sabit protezde 4 yıllık kullanım sonunda estetik, yumuşak doku reaksiyonları ve hasta memnuniyeti yüksek olmakla beraber \%31.25 oranında seramik ciping gözlenmiştir. ${ }^{13}$

\section{Monolitik zirkonya seramik sabit} protezler

Zirkonya (Zr02), biyouyumlu, biyoinert, radyoopak ve korozyon, eğilme ve kırılmaya karşı yüksek dirençli bir materyaldir. ${ }^{1}$ Monolitik/monoblok zirkonya olarak adlandırılan günümüzde geliştirilen 
seramik sistemleri zirkonya kor seramiklerin teknolojik versiyonlarıdır. Partikül boyutlarının (yaklaşık 40nm) düşük olması nano teknoloji ile üretilmesi, alümina içeriğinin azaltılması ile materyal yeni jenerasyon olarak kabul edilmektedir. Bilgisayar destekli tasarım ve bilgisayar destekli üretim teknolojisi kullanılarak tek parça seramik bloktan restorasyon yapılmaktadır. ${ }^{51}$ Geleneksel zirkonya kor materyalde gözlenen dezavantajların pek çoğu ortadan kalkmıştır. ${ }^{52}$ Avantajları; tek tabakalı bir sisteme izin vermesi, renk tabakaları bulunması ile estetik özelliklerinin artması, politür, glazür ve boyama işlemlerinin yapılması, antogonist mine için aşındırma-dostu olması, semi-translusent olması ile ön bölgede kullanılabilmesi, eğilme direncinin zirkonya kor (900-1 $000 \mathrm{Mpa}$ ) materyalden yüksek olması (1 100-1 $300 \mathrm{Mpa}$ ) hem ön hem de arka bölgede estetik ve dayanıklılık bakımından güvenle kullanılması, anterior ve posterior restorasyonlarda toplam kırılma oranının \%1.09 olması, 0.6-0.8 mm kalınlıkta yapılabilmesi, non adeziv ve/veya adeziv rezin yapıştırma simanları ile yapıştırılması, düşük ISı iletkenliği, kimyasal olarak stabil olması ve maliyetinin kısmen düşük olmasıdır. ${ }^{53-58}$

Bazı çalışmalarda bildirilen translusensi özelliğinin yeterli olmadığı, bu nedenle özellikle estetik bölgelerde üst yapı seramik materyali ile birlikte kullanılması gerektiği dezavantajıdır. ${ }^{12,20}$ Diğer dezavantajları ise, üst yapı materyali kullanılmadığı zaman doğrudan ağız sıvıları ile temasta olan monolitik zirkonyada düşük ısı bozunması olabileceği, pürüzlü yüzeylerin oklüzal aşındırma miktarını arttırdığı şeklinde sayılmaktadır. ${ }^{20,47}$ Ön bölge resstorasyonlarında kırılma oranının arka bölgeden daha yüksek olduğu ve çok üyeli köprü restorasyonlarda kırılma oranının tek tam kronların iki katı olduğu rapor edilmiştir. ${ }^{54}$

Tam dişsiz hastaların rehabilitasyonlarında, tam ark implant-destekli sabit dental monolitik zirkonya protezlerin yapılması ile ilgili kısa dönem verilere dayalı olan ve hasta bazlı klinik çalışmaların sonuçlarının değerlendirildiği bir derlemede kısa dönem başarılarının yüksek olduğu rapor edilmiştir. ${ }^{12,20,47} \mathrm{Bu}$ tedavilerin yaygın kullanımları için daha uzun süreli çalışmaların yapılması gerektiği belirtilmiştir. ${ }^{7} \mathrm{Bu}$ tür protezlerde monolitik zirkonya, vertikal ve horizantal rezorpsiyona bağlı gelişen pembe estetik gereksinimini karşılamaktadır. Pembe renkte olan zirkonya ile stabil ve doğal görünümlü pembe dişeti uygulması yapılmaktadır. $^{2}$ Bu tip restorasyonlar ile ilgili uzun dönem klinik çalışmalar bulunmazken kısa dönem çalışmalar ve klinik vaka raporları bulunmaktadır. ${ }^{59}$ Alt çene implantdestekli tam ark vida-tutuculu monolitik zirkonya bir protezde 18 aylık gözlem sonunda implant bağlantı bölgesinden restorasyonun kırıldığı ve tamir edilmesinin mümkün olmadığı belirtilmiştir. Ayrıca aşırı stres gelen implant çevresi bölgelerde bu tip protez planlamasında dikkat edilmesi gerektiği, kantilever uzunluğu fazla ve implant sayısı az olan vakalarda altın veya titanyum alt yapıların kullanımı önerilmiştir. ${ }^{59}$

Tam-ark implant-destekli monolitik zirkonya sabit protezlerin avantajları

1. Mekanik özellikleri metal alaşımlardan daha yüksektir. ${ }^{57}$

2. Yoğunluğu $6.1 \mathrm{~g} / \mathrm{cm}^{3}$ olduğu ve metal alaşımlardan 2-3 kez düşük olduğu için tamalanmış protez metal-seramik restorasyonlardan daha hafif olmaktadır. ${ }^{60}$

3. Titanyum alaşımlarında yoğunluk $4.5 \mathrm{~g} / \mathrm{cm}^{3}$ olmakla birlikte, titanyum alaşımı-akrilik protezlerde akrilik-titanyum bağlantı problemleri oluşmaktadır. ${ }^{60}$

4. Yüzey pürüzlülüğü, bakteri kolonizasyonu ve yüzey renklenmesi bakımından çok önemlidir. ${ }^{61}$ Akrilik rezinlerde bakteri tabakasının kalınlığı ortalama 0.03 - $1.2 \mu \mathrm{m}$ iken glazür yapılmış seramiklerde $0.08-0.33 \mu \mathrm{m}$, saf titanyum için $0.008-0.02 \mu \mathrm{m}$ ve zirkonya kor seramiklerde $0.08-0.1 \mu \mathrm{m}$ olması nedeni ile bakteriyel kolonizasyonun azaltılmasında uygun bir materyaldir. ${ }^{2}$

5. Karşıt diş aşındırması geleneksel feldspatik seramiklerden daha düşüktür. ${ }^{62,63}$

6. İmplantlar üzerine uyumunun nettir. ${ }^{39}$

7. Minimal oklüzal düzeltme gerektirir. ${ }^{39}$

8. Bu teknolojik protezler ile implant-destekli vidatutuculu ve/veya simante sabit protezler yapılabilmektedir. ${ }^{64}$

9. Monolitik zirkonya üzerine uygulanan seramiklerde oluşabilecek defektleri önlemek için preslenen cam seramik veneerlerde uygulanabilmektedir. ${ }^{8,65,66}$

10. Vida-tutuculu implant destekli sabit protezler yapılabilir. Böylece yumuşak dokuların daha sağlıklı olması, gerektiği zaman sökülebilmesi ve yer darlığı vakalarında kullanılması sağlanır. ${ }^{67}$

11. Siman-tutuculu implant destekli sabit protezler yapılabilir. Böylece, ideal oklüzal uyum, estetik, pasiflik ve retansiyon kaybının daha az olması sağlanır. ${ }^{67}$ 
Tam-ark implant-destekli monolitik zirkonya sabit protezlerin dezavantajları

1. Uzun dönem klinik destekli çalışma bulunmaması, ${ }^{64}$

2. 3-24 aylık takipte başarılı iken, seramik restorasyonlarda klinik olarak 5 yıllık kullanımda \%95 başarı gerekliliği bakımından çalışma olmaması, ${ }^{54,67}$

3. Fasiyel veneerlerde ciping oluşabilmesi, ${ }^{64}$

4. Translusensi özelliğinin cam seramiklerden düşük olmasıdır. ${ }^{68}$

Venezia ve ark ${ }^{64}$ retrospektif bir çalışmada 26 hastaya yapılan tek parça halinde, komple ark implantdestekli monolitik zirkonya protezlerde zirkonya üzerine fasiyel feldspatik seramik veneer uygulamışlardır. Simante protezlerde siman artıklarını temizlenme dezavantajlarına bağlı gelişebilecek olan periimplantit ve mukozit oluşmasını önlemek amacı ile vidalı protezler yapılmışıır. ${ }^{64} 20$ aylık gözlem sonunda hem implantlarda, hem de protezlerde sağ kalım oranı $\% 100$ implantlar da başarı oranı \%94 olarak verilmiştir. Sadece birkaç protezde anterior minör porselen ciping gözlenmiştir. ${ }^{64}$ Vida tutuculu implant-destekli protezlerin sökülmesinin kolay olmasına bağlı olarak mekanik ve biyolojik komplikasyon oranları daha azalmaktadır. Avrupa Osseointegrasyon Birliği (European Association of Osseointegration), çok sayıda implant destekli tedavilerde vida-tutuculu altyapıları önermektedir. ${ }^{48}$

Carames ve ark $^{39}$ tarafindan yapılan retrospektif vaka serisinde 14 hastaya yapılan 26 tam ark implant destekli restorasyonun klinik avantajları ve sınırlamaları rapor edilmiştir. 3-24 aylık gözlem sonunda implant sağ kalım oranı $\% 100$ ve monolitik zirkonya restorasyonlarda başarı oranı \% 96 olarak bulgulanmıştır. ${ }^{39}$ Fonksiyon, fonasyon, estetik ve hasta memnuniyeti bakımlarından başarıı restoras- yonlar olarak kabul edilmiştir. ${ }^{39}$ Bilgisayar destekli fasiyel kazıma yapılan (digital cut-back) monolitik zirkonya restorasyonlarda yüzey pürüzlülüğü minimal olduğu için 1 yıllık takip sonucunda sadece bir vakada anterior ciping gözlenmiştir. ${ }^{39}$

Vaka raporlarına bakıldığı zaman; iki hastaya konvansiyonel yükleme ile yapılan maksiller-mandibuler vida-tutuculu implant destekli monolitik zirkonya köprülerin 1 ylllık takibinde herhangi bir komplikasyona rastlanmamışır. ${ }^{12,16} 1$ hastaya immediyat yükleme ile yapılan mandibuler tam ark monolitik zirkonya köprünün 1 ylllık takip sürecinde herhangi bir sorun oluşmamıştır. ${ }^{69}$ Bir hastaya maksiller 8 implant üzerine yapılan tam ark monolitik zirkonya sabit protezde 1 yıllık kullanımda herhangi bir problem olmadığı belirtilmiştir. $^{2} 17$ hastaya yapılan mandibuler tam ark implant destekli monolitik zirkonya sabit protezlerde 1 yıllık takip sonuçlarına göre implant başarısı \%94 ve protez başarı oranı $\% 88$ olarak verilmiştir. ${ }^{6} 1$ hastaya yapılan maksiler vida-tutuculu protezde 3 yıl sonunda herhangi bir olumsuzluk gözlenmemiştir. ${ }^{5}$

\section{SONUC}

Tam dişsizlikte protetik tedavide CAD/CAM teknolojisi ve modern monolitik seramik materyaller ümit verici tedavi yaklaşımları sunmaktadır. Tam ark implant üstü monolitik zirkonya seramik köprü protezleri ile 5-10 yıl arası kısa dönemlerde, klinik, estetik ve dayanıklılık bakımından oldukça yüksek başarı oranına sahip sonuçlar elde edilebilmektedir. Ancak 10 yıl sonrası olarak değerlendirilen uzun dönem klinik başarı oranlarına ait yeterli veri bulunmadığı için, daha fazla çalışmaya gereksinim olduğu ve kesin bir sonuca ulaşmak için bulguların yeterli olmadığı gözlenmektedir.

Monolitik zirkonya seramik sistemlerin son yıllarda üretilen ve giderek gelişmekte olan bilgisayar destekli seramik sistemler olmaları nedeniyle, tam ark implant üstü restorayonların yapımında uzun dönem klinik ve in vitro çalışmaların yapılması ve yeni uygulama tekniklerinin geliştirilmesine intiyaç duyulmaktadır.

Rukiye Durkan: ORCID ID: 0000- 0002-3381-4073

Gonca Deste: ORCID ID: 0000-0002-5481-0063

Perihan Oyar: ORCID ID: 0000-0003-3849-9153

\section{KAYNAKLAR}

1. Tulunoğlu İF. Günümüz Dişhekimliğinde İmplantoloji .Üçüncü baskı, Ankara 2011， s. 615.

2. Sadid-Zadeh R, Liu PR, Aponte-Wesson R, O'Neal SJ. Maxillary cement retained implant supported monolithic zirconia prosthesis in a full mouth rehabilitation: a clinical report. J Adv Prosthodont 2013;5:209-17.

3. Larsson C, von Steyern PV. Implant-supported fullarch zirconia-based mandibular fixed dental prostheses. Eightyear results from a clinical pilot study. Acta Odontol Scand 2013;71:1118-22.

4. Papaspyridakos P, Lal K. Computer-assisted 
design/computerassisted manufacturing zirconia implant fixed complete prostheses: clinical results and technical complications up to 4 years of function. Clin Oral Impl Res 2013; 24: 659-65.

5. Thalji GN, Cooper LF. Implant-supported fixed dental rehabilitation with monolithic zirconia: A clinical case report. J Esthet Restor Dent 2014;26:88-96.

6. Limmer B, Sanders AE, Reside G, Cooper LF. complications and patient-centered outcomes with an Implant-supported monolithic zirconia fixed dental prosthesis: 1 year results. J Prosthod 2014; 23: 267-75.

7. Abdulmajeed AA, Lim KG, Närhi TO, Cooper LF. Complete-arch implant-supported monolithic zirconia fixed dental prostheses: A systematic review. J Prosthet Dent 2016;115:672-7.

8. Rojas-Vizcaya. Full zirconia fixed detachable implant-retained restorations manufactured from monolithic zirconia: clinical report after two years in service. J Prosthodont 2011;20:570-6.

9. Guess PC, Att W, Strub JR. Zirconia in fixed implant prosthodontics. Clin Implant Dent Related Res 2012;14:633-45.

10. Kanat B, Cömlekoğlu EM, Dündar-Çömlekoğlu $M$, Hakan Sen B, Ozcan M, Ali Güngör M. Effect of various veneering techniques on mechanical strength of computer-controlled zirconia framework designs. J Prosthodont 2014;23:445-55.

11. Balshi TJ, Wolfinger GJ, Slauch RW, Balshi SF. A retrospective analysis of 800 Brånemark System implants following the all-on-four protocol. J Prosthodont 2014;23:83-8.

12. Altarawneh S, Limmer B, Reside GJ, Cooper L. Dual jaw treatment of edentulism using implantsupported monolithic zirconia fixed prostheses. J Esthet Restor Dent 2015;27:63-70.

13. Rohlin M, Nilner K, Davidson T, Gynther G, Hultin $M$, Jemt T, Lekholm $U$, Nordenram G, Norlund A, Sunnegardh-Gronberg K, Tranaeus S. Treatment of adult patients with edentulous arches: a systematic review. Int J Prosthodont 2012;25:553-67.

14. Patzelt SB, Spies BC, Kohal RJ. CAD/CAMfabricated implant-supported restorations: a systematic review.Clin Oral Implants Res 2015;26:77-85.

15. Anusavice KJ, Shen C, Rawls HR. Phillips' science of dental materials. 12th ed. Philadelphia:
Saunders/Elsevier; 2013, p. 291-451.

16. Mehra M, Vahidi F. Complete mouth implant rehabilitation with a zirconia ceramic system: A clinical report. J Prosthet Dent 2014;112:1-4.

17. Mertens C, Steveling HG. Implant-supported fixed prostheses in the edentulous maxilla: 8-year prospective results. Clin Oral Impl Res 2011; 22:464-72.

18. Wittneben JG, Millen C, Brägger U. Clinical performance of screw- versus cement-retained fixed implant-supported reconstructions-a systematic review. Int J Oral Maxillofac Implants 2014;29:84-98.

19. Leempoel PJ, Van Rossum GM, de Hann AF. Survival studies of dental restorations: criteria, methods and analyses. J Oral Rehabil 1989;16:387-94.

20. Alani A, Maglad A, Nohl F. The prosthetic management of gingival aesthetics. $\mathrm{Br}$ Dent $\mathrm{J}$ 2011;210:63-9.

21. Linkevicius T, Vladimirovas E, Grybauskas S, Puisys A, Rutkunas V. Veneer fracture in implantsupported metal-ceramic restorations. Part I: Overall success rate and impact of occlusal guidance. Stomatologija 2008;10:133-9.

22. Roberts $\mathrm{DH}$. The failure of retainers in bridge prostheses. An analysis of 2,000 retainers. Br Dent J 1970;128:117-24.

23. Jacobi R, Shillingburg HT Jr, Duncanson MG Jr. Effect of abutment mobility, site, and angle of impact on retention of fixed partial dentures. ] Prosthet Dent 1985;54:178-83.

24. Ehrenkranz H, Langer B, Marotta L. Complete-arch maxillary rehabilitation using a custom-designed and manufactured titanium framework: a clinical report. J Prosthet Dent 2008;99:8-13.

25. Llobell A, Nicholls JI, Kois JC, Daly CH. Fatigue life of porcelain repair systems. Int J Prosthodont 1992;5:205-13.

26. Reuter JE, Brose MO. Failures in full crown retained dental bridges. Br Dent J 1984;157:61-3.

27. Priest G, Smith J, Wilson MG. Implant survival and prosthetic complications of mandibular metalacrylic resin implant complete fixed dental prostheses. J Prosthet Dent 2014;1:466-75.

28. Cox JF, Zarb GA. The longitudinal clinical efficay of osseointegrated dental implants: a 3-year report. Int J Oral Maxillofac Implants 1987;2:91-100. 
29. Gallucci GO, Doughtie CB, Hwang JW, Fiorellini JP, Weber HP. Five-year results of fixed implantsupported rehabilitations with distal cantilevers for the edentulous mandible. Clin Oral Impl. Res 2009;20:601-7.

30. Kwon T, Bain PA, Levin L. Systematic review of short- (5-10 years) and longterm (10 years or more) survival and success of full-arch fixed dental hybrid prostheses and supporting implants. J Dent 2014;42:1228-41.

31. Real-Osuna J, Almendros-Marques N, Gay-Escoda C. Prevalence of complications after the oral rehabilitation with implant-supported hybrid prostheses. Medicina Oral 2012:116-21.

32. Branemark PI, Svensson B, van Steenberghe D. Ten-year survival rates of fixed prostheses on four or six implants ad modum Branemark in full edentulism. Clin Oral Implants Res 1995;6:227-31.

33. Papaspyridakos $\mathrm{P}$, Chen $\mathrm{CJ}$, Chuang SK, Weber HP, Gallucci GO. A systematic review of biologic and technical complications with fixed implant rehabilitations for edentulous patients. Int J Oral Maxillofac Implants 2012;27:102-10.

34. Purcell BA, McGlumphy EA, Holloway JA, Beck FM. Prosthetic complications in mandibular metal-resin implant-fixed complete dental prostheses: a 5- to 9-year analysis. Int $\mathrm{J}$ Oral Maxillofac Implants 2008;23:847-57.

35. Bozini T, Petridis H, Garefis K, Garefis P. A metaanalysis of prosthodontic complication rates of implant-supported fixed dental prostheses in edentulous patients after an observation period of at least 5 years. Int $J$ Oral Maxillofac Implants 2011;26:304-18.

36. Afrashtehfar KI. The all-on-four concept may be a viable treatment option for edentulous rehabilitation. Evid Based Dent 2016;17:56-7.

37. Jemt T. Failures and complications in 391 consecutively inserted fixed prostheses supported by Brånemark implants in edentulous jaws: a study of treatment from the time of prosthesis placement to the first annual checkup. Int J Oral Maxillofac Implants 1991;6:270-6.

38. Ortorp A, Jemt T. Clinical experiences of CNCmilled titanium frameworks supported by implants in the edentulous jaw: 1-year prospective study. Clin Imp Dent Rel Res 2000;2:2-9.
39. Carames J, Suinaga LT, Yu YC, Pérez A, Kang M. Clinical advantages and limitations of monolithic zirconia restorations full arch Implant supported reconstruction: Case Series Int J Dent 2015; 2015: 392496

40. Fischer K, T Stenberg. Prospective 10-year cohort study based on a randomized, controlled trial (RCT) on implant-supported full-arch maxillary prostheses. Part II: prosthetic outcomes and maintenance. Clin Implant Dent Related Res 2013;15:498-508.

41. Bergendal B, Palmqvist S. Laser-welded titanium frameworks for implant-supported fixed prostheses: a 5-year report. Int J Oral Maxillofac Implants 1999;14:69-71.

42. Ghazal M, Hedderich J, Kern M. Wear of feldspathic ceramic, nano-filled composite resin and acrylic resin artificial teeth when opposed to different antagonists. Eur J Oral Sci 2008;116:58592.

43. Coachman C, Salama M, Garber D, Calamita M, Salama $H$, Cabral $G$. Prosthetic gingival reconstruction in fixed partial restorations. Part 3: laboratory procedures and maintenance. Int $\mathrm{J}$ Periodontics Restorative Dent 2010;30:19-29.

44. Koizuka M, Komine F, Blatz MB, Fushiki R, Taguchi $K, H$ Matsumura The effect of different surface treatments on the bond strength of a gingivacolored indirect composite veneering material to three implant framework materials Clin Oral Impl Res 2013;24:977-84.

45. Al-Amleh B, Lyons K, Swain M. Clinical trials in zirconia: a systematic review. J Oral Rehabil 2010;37:641-52.

46. Karaalioğlu OF, Duymuş Z Y. Diş hekimliğinde uygulanan CAD/CAM sistemleri. Atatürk Üniv Diş Hek Fak Derg 2008;18:25-32.

47. Raigrodski AJ, Chiche GJ, Potiket N, Hochstedler $\mathrm{JL}$, Mohamed SE, Billiot S. The efficacy of posterior three-unit zirconium-oxide-based ceramic fixed partial dental prostheses: a prospective clinical pilot study. J Prosthet Dent 2006;96:237-44.

48. Sailer I, Feher A, Filser F, Gauckler LJ, Lüthy $H$, Hämmerle $\mathrm{CH}$. Five-year clinical results of zirconia frameworks for posterior fixed partial dentures. Int J Prosthodont 2007;20:383-8.

49. Stefan $\mathrm{H}$, Michael B, Enrico S, Markus BB, Manfred $W$. The application of zirconium oxide frameworks

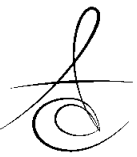


for Implant superstructures. Quintessence Dent Tech 2006;29:103-12.

50. Bayramoğlu E, Özkan Y. Cam seramik restorasyonlar ve zirkonya alt yapılı seramik restorasyonların karşılaş̧ırılması. Atatürk Üniv. Diş Hek. Fak. Derg. 2012;6:110-23.

51. Ueda K, Güth JF, Erdelt K, Stimmelmayr $M$, Kappert $H$, Beuer $F$. Light transmittance by a multicoloured zirconia material. Dent Mater J 2015;34:310-4.

52. Zhang $F$, Vanmeensel $K$, Batuk M, Hadermann J, Inokoshi M, Van Meerbeek B, Naert I, Vleugels J. Highly-translucent, strong and aging-resistant $3 Y$ TZP ceramics for dental restoration by grain boundary segregation. Acta Biomater 2015;16:215-22.

53. Sulaiman $T A$, Abdulmajeed AA, Donovan TE, Ritter AV, Vallittu PK, Närhi TO, Lassila LV. Optical properties and light irradiance of monolithic zirconia at variable thicknesses. Dent Mater 2015;31:1180-7.

54. Sulaiman $T A$, Abdulmajeed AA, Donovan $T E$, Cooper LF, Walter R. Fracture rate of monolithic zirconia restorations up to 5 years: A dental laboratory survey. J Prosthet Dent 2016:10.1016.

55. Inokoshi M, De Munck J, Minakuchi S, Van Meerbeek B. Meta-analysis of bonding effectiveness to zirconia ceramics. J Dent Res 2014;93:329-34.

56. Tinschert J, Natt G, Mohrbotter N, Spiekermann H, Schulze KA. Lifetime of alumina-and zirconia ceramics used for crown and bridge restorations. J Biomed Mater Res B Appl Biomater 2007;80:31721.

57. Schärer P. All-ceramic crown systems: clinical research versus observation in supporting claims. Signature 1997;4:1.

58. Tiu J, Al-Amleh B, Waddell JN, Duncan WJ. Clinical tooth preparations and associated measuring methods: a systematic review. J Prosthet Dent 2015;113:175-84.

59. Chang JS, Ji W, Choi CH, Kim S. Catastrophic failure of a monolithic zirconia prosthesis: Clinical Report. J Prosthet Dent 2015;113:86-90.

60. Ronald LS, John MP. In: Evolve resources for Craig's restorative dental materials. 12th ed. Mosby: 2006.

61. Bollen CM, Lambrechts P, Quirynen M. Comparison of surface roughness of oral hard materials to the threshold surface roughness for bacterial plaque retention: a review of the literature. Dent Mater 1997;13:258-69.

62. Preis V, Behr M, Handel G, Schneider-Feyrer S, Hahnel $S$, Rosentritt $M$. Wear performance of dental ceramics after grinding and polishing treatments. J Mech Behav Biomed Mater 2012;10:13-22

63. Denry I, Kelly JR. State of the art of zirconia for dental applications. Dent Mater 2008;24:299-307.

64. Venezia P, Torsello F, Cavalcanti R, D'Amato S. Retrospective analysis of 26 complete-arch implant-supported monolithic zirconia prostheses with feldspathic porcelain veneering limited to the facial surface. J Prosthet Dent 2015;114:506-12

65. Ishibe M, Raigrodski AJ, Flinn BD, Chung KH, Spiekerman $C$, Winter RR. Shear bond strengths of pressed and layered veneering ceramics to highnoble alloy and zirconia cores. J Prosthet Dent 2011;106:29-37.

66. Rosentritt M, Preis V, Behr M, Hahnel S, Handel G, Kolbeck C. Two-body wear of dental porcelain and substructure oxide ceramics. Clin Oral Investig 2012;16: 935-43.

67. Sherif S, Susarla HK, Kapos T, Munoz D, Chang BM, Wright RF. A systematic review of screwversus cement-retained implant-supported fixed restorations. J Prosthodont 2014;23:1-9.

68. Ilie N, Stawarczyk B. Quantification of the amount of blue light passing through monolithic zirconia with respect to thickness and polymerization conditions. J Prosthet Dent 2015;113:114-21.

69. Cheng CW, Chien $\mathrm{CH}, \mathrm{CJ}$ Chen, Papaspyridakos P. Complete-mouth implant rehabilitation with modified monolithic zirconia implant-supported fixed dental prostheses and an immediate-loading protocol: A clinical report. J Prosthet Dent 2013;109:347-35.

\section{Yazışma Adresi}

Doç. Dr. Rukiye Durkan, Afyon Kocatepe Üniveritesi Diş Hekimliği Fakültesi, Protetik Diş Tedavisi Anabilim Dalı, Afyon e-mail:dr.durkan@hotmail.com 